\title{
Coil geometry models for power loss analysis and hybrid inductive link for wireless power transfer applications
}

\author{
CHANDRASEKHARAN NATARAJ ${ }^{1, *(D)}$, SHEROZ KHAN ${ }^{2}$ and MOHAMED HADI HABAEBI ${ }^{2}$ \\ ${ }^{1}$ Faculty of Computing, Engineering and Technology, Asia Pacific University of Technology and Innovation, \\ Kuala Lumpur, Malaysia \\ ${ }^{2}$ Department of Electrical and Computer Engineering, International Islamic University Malaysia (IIUM), \\ Kuala Lumpur, Malaysia \\ e-mail: chander@apu.edu.my; sheroz@iium.edu.my; habaebi@iium.edu.my
}

MS received 27 April 2017; revised 4 January 2018; accepted 18 January 2018; published online 26 April 2018

\begin{abstract}
This paper presents a hybrid inductive link for Wireless Power Transfer (WPT) applications. Achieving better power transfer efficiency over a relatively wider distance across coils is the prime objective in most of the WPT systems, but often suffers from power loss in the near field area of inductively coupled coils. One of the reasons for this power loss is the pattern of the magnetic field produced by the source coil used in the WPT system. Mostly the nature of magnetic field produced by the source coil is distributed radially over the coil, in which the produced magnetic field is not fully utilized. Achieving better efficiency and load current by reducing power loss is the main driving force of this work. One of the viable methods to reduce the power loss is by increasing the field intensity thereby redirecting the flux lines flow to be directional. With this aim, three coils such as solenoid, spiral and conical are designed and simulated to determine the magnetic field strength using Finite Element Method. The conical coil produces the highest self-inductance of $8.63 \mu \mathrm{H}$ and a field strength of $1.542 \mathrm{~Wb}$ with the coil thickness of $3.20 \mathrm{~mm}$. Then, WPT system is demonstrated with the inclusion of Maximum Power Point Tracking algorithm for improving efficiency. The schematic of flux generation of both in the transmitter and receiver sections are demonstrated and analyzed graphically. The efficiency of both simulation and experimental measurements are matched well with similar progression. The effect of parameters (angle, distance, and load resistance) on the efficiency is explored. The outcomes conclude that the inductive coupling has achieved $73 \%$ (average case) power transfer wirelessly over a distance of $5 \mathrm{~cm}$ with an input voltage of $5 \mathrm{~V}$ and $5 \mathrm{MHz}$ frequency.
\end{abstract}

Keywords. Coils geometry; inductive coupling; MPPT algorithm; power transfer efficiency; resonant coils; wireless power transfer.

\section{Introduction}

Inductively Coupled Wireless Power Transfer (ICWPT) has been used as the main contender with other WPT techniques after a long journey since Nikola Tesla explored its idea for the first time in 1904. In the past two decades, it was, unfortunately, getting much less attention on the consumer market mainly due to technology limitations. Years ago, no one imagined it to be convenient and so much affordable. As of today when we find much freedom and flexibility to adopt it. Now it seems as a paramount reporting in the research and publications of the wireless power transfer technologies.

ICWPT offers a simple and compact inductive coupling based approach. It consists of two coils placed narrow-gap apart, which is able to transport the power from one coil to

*For correspondence another coil through inductive coupling. When the source coil is excited with pulsed power, then the load-coil receives it to power up the load. It removes altogether the need for wiring accessibility. It has been reported for use in both implantable and non-implantable applications. In the biomedical monitoring applications, several batteries operated sensors are implanted in a patient's body to evaluate the biophysical profile through observing body temperature [1], blood-sugar level [2], and blood pressure [3]. The power supply to such embedded devices is of major concern, as it is not practical to use batteries, which are exhaustible and carries the hazard of chemical leakage.

The option-list of using wireless power transfer system is endless. Most of the electronic devices such as cellphones, tablets, and laptop computers are battery operated, which needs to be recharged very often. Larger power demanding systems like Robots and Electric Vehicles use inductively coupled-based WPT which supports significantly in terms of 
safe charging embedded with on-board power management features. Batteries as reliable power sources in all our portable electronic devices, which are losing ground due to the nature of slow charging, limited energy, weight, and cost. It is essential that ICWPT should offer the viable solutions, thus meeting the battery technology limitations [4-6]. ICWPT are proving the most used ones in charging traction batteries of electric vehicles in the automobile field and also domestic induction cooking appliances [7]. The applications involving solar cells, lasers, microwaves and resonance of electromagnetic waves have had the latest success in the field of WPT [8, 9]. This technology finds realistically viable applications in the area of Distribution Generation (DG) system [10]. On the other hand, circuits such as RFID tags, embedded sensors, and buried devices, work at low voltage range, because of the power requirements and its restrictions. The portable devices operated particularly with low voltage, brings some inherent problems such as ripples and spikes of reliability concerns [11]. Among the different applications, the presence of similar power level constraint generates strong interest in probing further into the wireless power technologies, using mostly impedance matching condition in ensuring effective power transfer.

Among the variety of power transfer modes offered for WPT, resonance based inductive coupling is the most popular and preferable technique. The selection of modes depends on the requirement of power level, operating frequency, size, transfer distance, and transfer power. Inductive power transfer system works more efficiently in near field (strongly coupled) applications compared to far-field (loosely coupled) ones [12, 13], where transfer distance and power is limited to within centimeters and tens of kilowatts, respectively. The working frequency of inductive coupling is in the $\mathrm{kHz}$ band and can be raised into $\mathrm{MHz}$ range by operating under resonance mode, supported with external capacitors [14]. Due to requirement of current electronic devices and ISM [Industrial Scientific and Educational] band compliant regulation, $\mathrm{MHz}$ band is usually desired [15]. Other applications like sensor networks, the multi-frequency operation is another criterion for which source coil must be intelligent enough to transfer enough power to the multiple loads efficiently $[16,17]$. The feasible solution is that to wind the coil with unique geometry, capable of dealing with a wider range of power requirement options. In applications of smaller receiver coil requirement, the expectancy on power density in the source coil becomes larger. Further expected technical challenge in such WPTs is alignment (orientation) of coils. Most of the cases are misalignment configuration whereas coils misaligned axially, or oriented by angle or both. Therefore, WPT also needs to be explored in the nonorientation environment [18].

Flux leakage in the coil vicinity is another design constraint which should not affect the human health when it is being deployed in real-time and hence emission of source coil field standards are compiled [19, 20]. By contrast, the interpretation of resonant coils used in the WPT system is very safe and will not make any biological effects in midrange applications. Therefore, magnetic resonance based WPT system is quite popular and commonly debated subject. Thus, the most effective geometrical configuration for efficient wireless power transfer makes a good reason for this work. Aside all, the power received at the receiving end is poor, which results in less power transferred and most of the power is wasted in the form of heat [21]. ICWPT system always suffers from power loss due to the nature of the emitted field, which is of distributive pattern in the coil vicinity [22]. To the best of authors' knowledge, power loss in the coil zone has been a severe limitation and so far limited works have been reported on this. One of the viable solutions is to redirect the distributive patterned field into a unidirectional; power loss can be controlled significantly. To this effect, details of our previous work have presented [23] using exclusive coil model along with analysis.

This paper explores experimentally power loss with misalignment analysis in a WPT with a typical transmitterreceiver coil configuration. Analyses of the coil geometries and significance of coil dimensions for power transfer efficiency is presented. First, the conical coil is developed for better magnetic field pattern using finite element simulation tool. Then, Matlab-based WPT environment is established using Maximum Power Point Tracking (MPPT) algorithm for efficiency analysis. Finally, it is validated through experimental results using developed coils under orientation and skewed configuration.

This paper is organized as consisting of five sections including the introduction. In section, the basic theory involved with WPT along with different coil dimensions are described in detail. Further, design and simulation of solenoid, spiral and conical coils using Solid Works with COMSOL are explored for field and power loss analysis in section 3. Section 4 describes the developed Matlab based WPT simulation platform and experimental set-up for efficiency analysis. Finally, the conclusion is drawn in Section 5 .

\section{System configuration and inductive link}

An overall block diagram of a typical Wireless Power Transfer (WPT) system is illustrated in figure 1. In the transmitter section, resonance based inductive coil driven by the DC input source is responsible for signal transmission. The preferable choice of operation is a resonant mode with less power loss, using high-quality factor coil, which should oscillate to its maximum amplitude. Besides the coil dimensions and shape, the operation of transmitter-receiver coil pair at similar resonant frequency assures maximum efficiency. The magnetic field with strong intensity generated by the source coil has been received by the resonantly coupled load coil in the receiver. Then the voltage is 


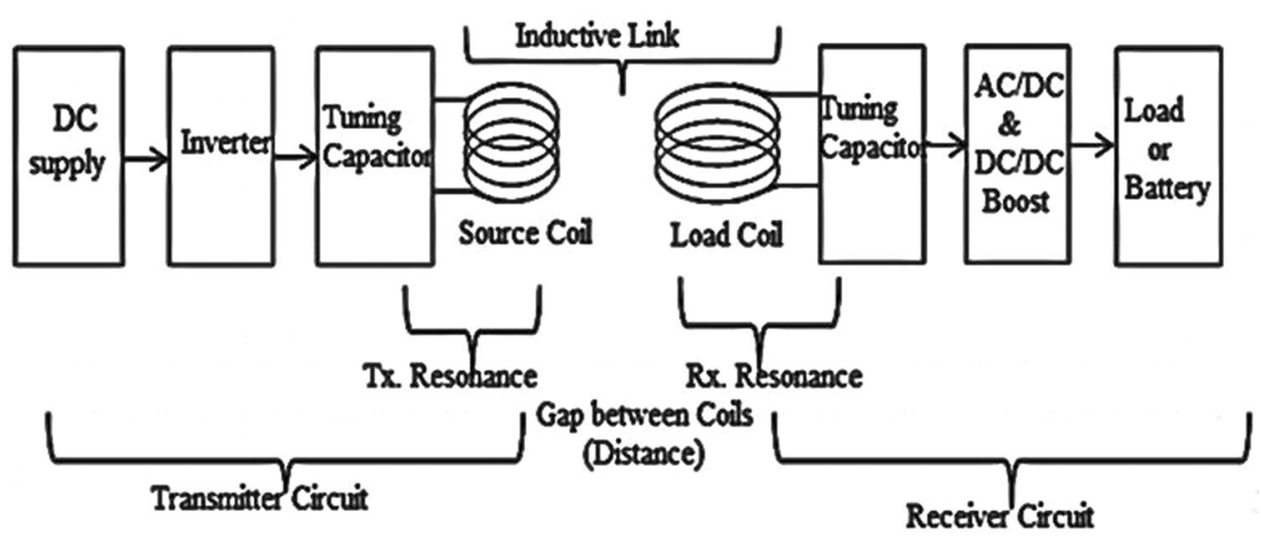

Figure 1. An overall block diagram of transmitter-receiver coil pair using resonant inductive coupling for wireless power transfer technique.

harvested in the receiver over a wider air-gap with minimum loss.

In order to ensure the compensation technique to reduce the stray and inter-turn capacitances, both coils must be tuned at the resonant frequency. The dc bias voltage is converted into AC pulses through an inverter, which would energize the inductively coupled source coil. Then, it transmits energy magnetically to the remotely located target load, to be powered or battery to be charged.

\subsection{Inductive link model}

The inductive link model is an electric equivalent circuit for the practical implementation of WPT system. It depicts the circuit level description of primary (or source) and secondary (or load) coil relationship, which is more abstractive. The general model used to describe the inductive link under consideration is as shown in figure 2.

In this inductive link, the parameter $R_{i n}$ represents the total input resistance of the source circuit. The values of $R_{p}$, $L_{p}$, and $C_{p}$ are the resistance, inductance, and capacitance of the transmitter coil. The values $R_{s}, L_{s}$, and $C_{s}$ are the resistance, inductance, and capacitance involved with the receiver coil. The parameter $k$ refers to the coupling factor present in the coupled zone. In most of the low-frequency WPT applications, the coils capacitance $C_{p}$ and $C_{s}$ are generally ignored. The source-load coil with $L_{p} C_{p}$ as well as $L_{s} C_{s}$ a tank are tuned at a common frequency. With the assumption of currents and voltages in r.m.s values, the system can be written as,

$$
\begin{aligned}
& {\left[\begin{array}{ll}
R_{p}+j\left(\omega L_{P}-\frac{1}{\omega C_{P}}\right) & -j \omega M \\
-j \omega M & R_{s}+j\left(\omega L_{s}-\frac{1}{\omega C_{s}}\right)
\end{array}\right]\left[\begin{array}{c}
I_{p} \\
I_{s}
\end{array}\right]} \\
& =\left[\begin{array}{c}
V_{p} \\
0
\end{array}\right]
\end{aligned}
$$

In the resonance condition, reactive parts of impedance are cancelled each other and power can be maximum. Then, the efficiency can be expressed as the ratio of harvested power at the load to the supplied power by the source [4].

$$
\eta=\frac{P_{L}}{P_{S}}=\frac{V_{L} I_{L} \cos \phi_{L}}{V_{S} I_{S} \cos \phi_{S}}
$$

For the condition of maximum power gained under resonance, the source power with current $I_{p}$ is written as,

$$
P_{p}=I_{p}^{2} \cdot R_{p}
$$

At the receiver side, the total impedance should be matched with the impedance $Z_{s}$ involved with load coil impedance for maximum power transfer.

$$
Z_{s}=R_{s}-j \omega L_{s}
$$

The real component of power at the secondary side via inductive coupling can be defined as,

$$
P_{s}=\frac{V_{s}^{2}}{R_{s}}
$$

Similarly, received power across the load coil in the secondary section is,

$$
P_{R}=\frac{V_{G e n}^{2}}{4 R_{L}}
$$

The magnetic field vector at the load coil can be determined through integrating Biot-Savart's law around the source coil which can be obtained as [5],

$$
H=\frac{I_{P}}{4 \pi} \oint \frac{d l \times r}{r^{3}}
$$

In Eq. (7), the strength of the field depends on the length, dimensions, and placement of source coil. By considering load coil as a closed loop, it can be solved using Faraday's law. 


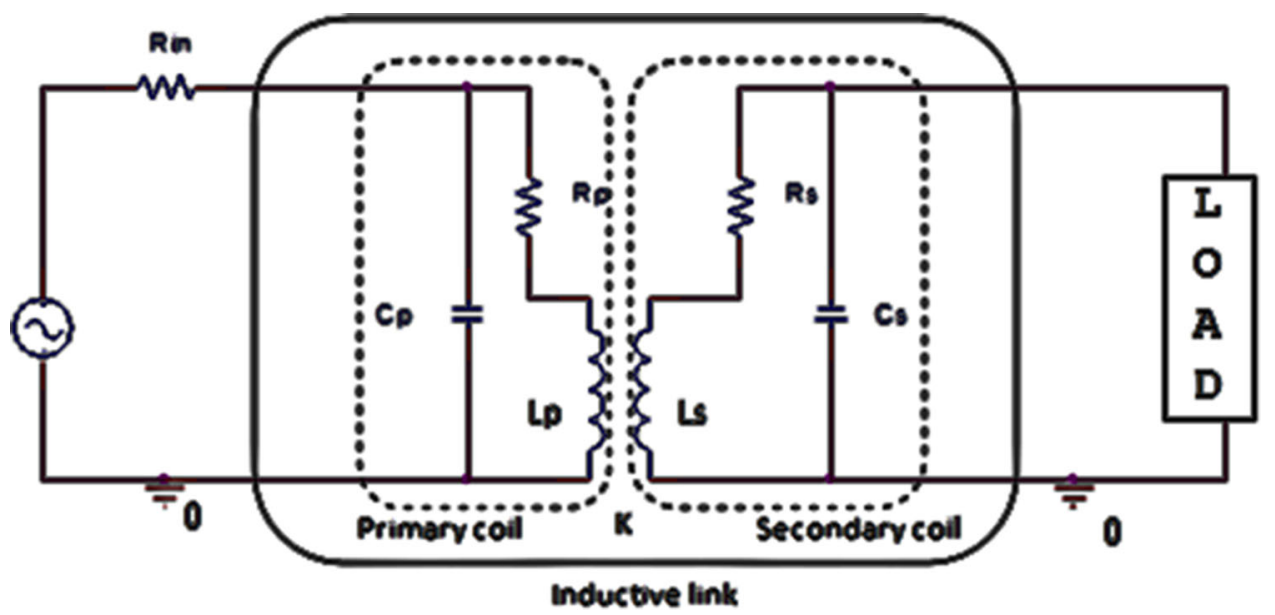

Figure 2. Typical inductive link model for WPT.

$$
V_{G e n}=-\frac{\partial}{\partial t} \int_{\mathbf{S}} B \cdot d S
$$

Which is the rate of change of flux (B) in the surface (S) of the coil. The appropriate form of Eq. (8) can be represented relating to the coil as follows [18]:

$$
V_{\text {Gen }}=\mu_{0} A_{L} j \omega H
$$

Where $A_{L}$ is an area of the load coil, $\mu_{0}$ is permeability of free space, and $H$ is a magnetic field strength calculated in (7). Then the power transfer efficiency for a pair of strongly coupled coils can be expressed as [20],

$$
\eta=\frac{\mu_{0}^{2} \pi^{2} N_{p}^{2} N_{s}^{2} a^{4} b^{4} \omega^{2}}{16 R_{p} R_{s}\left(a^{2}+d^{2}\right)^{3}}
$$

It is obviously seen that the efficiency depends on the number of turns, coil radius, the spacing between turns and coil resistances.

Apart from coil radii, spacing, and resistance, a number of turns used in the coils are directly proportional to efficiency. The measurement of coupling factor with respect to a number of turns is shown in figure 3. The spiral and conical coils are simulated to extract the values and verified with theoretical concepts. When the number of turns in the coil increases, coupling factor $(\mathrm{k})$ also gets increased and then better efficiency is attained. In conjunction with this, analysis of load coil geometry is conducted to find the optimum load coil geometry through the fixed source coil. The load coil profile to be used in the system is in the form of circular, rectangular and conical.

Figure 4 illustrates the output voltage and efficiency measurement of variously sized spiral shaped load-side coils. The geometry of load coil is analyzed through the coil configuration of spiral shaped load coil excited through a conical source coil with the height of $242 \mathrm{~mm}$

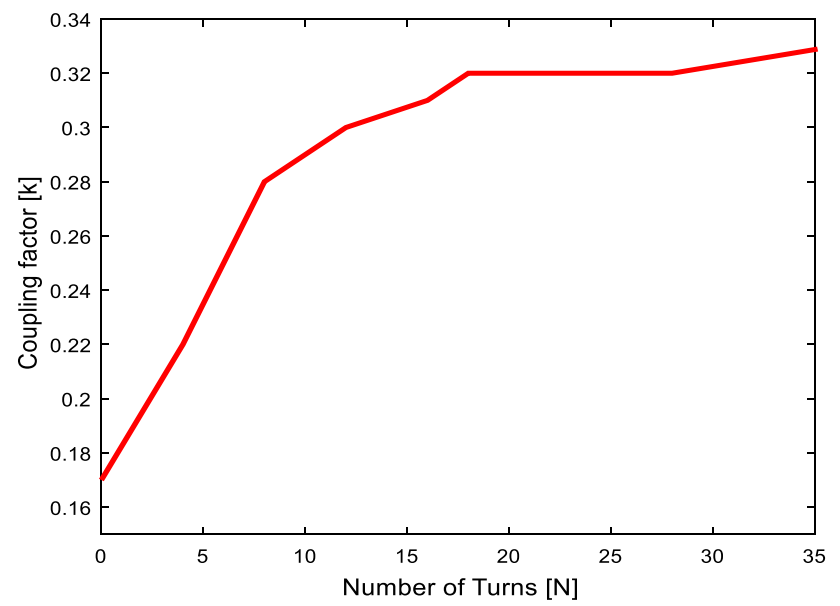

Figure 3. Coupling factor $(\mathrm{k})$ as a function of a number of turns for maximizing the transfer efficiency.

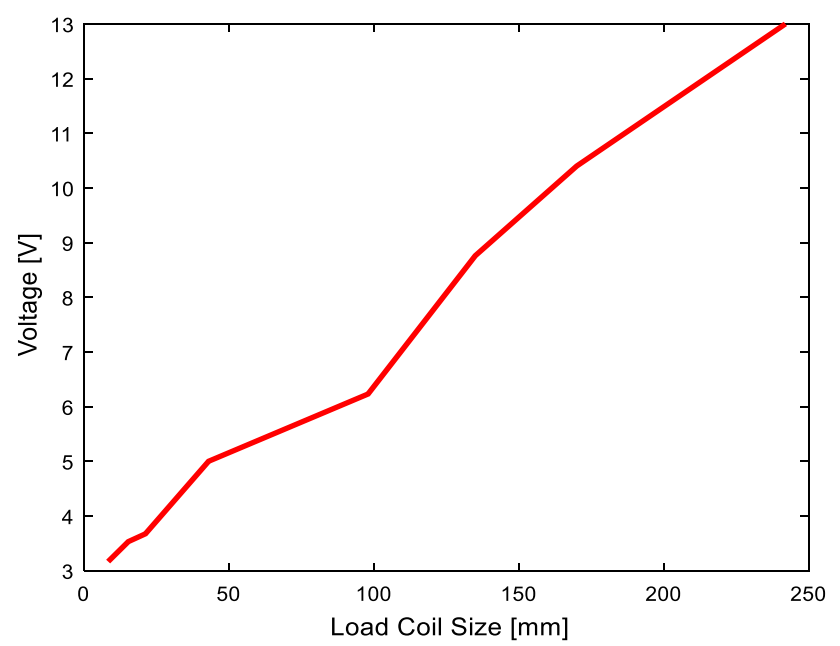

Figure 4. Measurement of the voltage at the load coil with respect to the coil size for maximizing the transfer efficiency. 
and supply voltage of $15 \mathrm{~V}$. Then, the size of the load coil is varied and measurements are analyzed accordingly. The load coils are placed at the bottom of the center axis of the source coil at a separation distance of $10 \mathrm{~cm}$ for strong inductive coupling.

It is observed that the maximum efficiency of $95 \%$ under the same sized coil scenario with proper orientations and the relative distance between coils is fixed at $5 \mathrm{~cm}$. Thus, it is concluded that the efficiency increases correspondingly with the increase of load coil geometry. Therefore, it is necessary to use a good number of turns for producing the sufficient voltage. It apparently depends on the geometry of source coil to generate the better magnetic field for energy harvesting applications.

\section{Coil structure design and finite element simulation}

The principle of power transfer through inductively coupled coils in most of the systems are governed by the relationship of field intensity and radiation pattern of the magnetic field around the coil. According to the Biot-Savart Law (13), the field intensity of load coil related to source coil in the vicinity of near field zone is governed by the two equations.

$$
\begin{aligned}
\vec{D} & =\varepsilon \vec{E} \\
\vec{B} & =\mu \vec{H}
\end{aligned}
$$

In the near field zone, maximum current flow is induced in the load coil through the flux linkage with source coil. The magnetic flux produced in a current carrying conductor can be written as,

$$
\vec{B}=\nabla \times \vec{A}(\vec{m})=\frac{\mu I}{4 \pi} \int\left(\nabla \times \frac{d \vec{l}}{\vec{m}}\right)=\frac{\mu I}{4 \pi} \int \frac{d \vec{l} \times \vec{m}}{\|m\|^{3}} .
$$

From Eq. (13), it is more significant to determine the path current $I$ in the single turn of the coil as a differential length element $d l$ with the distance vector $m$ between $d l$ and point of interest. These values are decided based on the geometrical parameters of the coil such as shape and dimensions. In order to determine the expression for $d l$, the geometry of solenoid, spiral, and helix patterned coils are defined using parametric curves in the subsequent sections. The coil geometries are defined using a different set of mathematical curves, which are then applied to the Biot-Savart Law for obtaining the expected field intensity. These geometrical parameters are contributed to determine the field pattern of coils and also to be optimized for obtaining the better transfer efficiency.

\subsection{Coil geometry of solenoid}

The geometry of a solenoid can be defined in a simple way of using mathematical equations supporting the circle in a plane, disregarding coil winding effects. In common, parameterized circle equations are used to define the solenoid geometry more accurately over the interval $[0,2 n \pi]$ as shown in the Eqs. (14.1)-(14.3).

$$
\begin{gathered}
x(t)=a \cos (t) \\
y(t)=a \sin (t) \\
z(t)=\frac{h}{2 \pi n} t .
\end{gathered}
$$

where $h$ is the height, $n$ is the number of turns and $a$ is the solenoid coil radius. The derivatives for finding incremental length vector $d l$ in the path of a three-dimensional solenoid. The relative equations of solenoid geometry are shown in the Eqns. (15a)-(15c).

$$
\begin{gathered}
x^{\prime}(t)=-a \sin (t) \\
y^{\prime}(t)=a \cos (t) \\
z^{\prime}(t)=\frac{h}{2 \pi n} .
\end{gathered}
$$

\subsection{Coil geometry - spiral}

As the same way of modeling the solenoid coil, parametric circle equations can be used to define the spiral coil inclusion with spiral Archimedes equations (uniform distance between spirals) over the interval $[0,2 n \pi]$. Neglecting the field distribution and inter-turn effects in the lateral direction, the relative equations for spiral geometry can be written as shown in the Eqns. (16.1)(16.2).

$$
\begin{aligned}
& x(t)=a \cos (t)+\frac{\delta}{2 \pi} t \cos (t) \\
& y(t)=a \sin (t)+\frac{\delta}{2 \pi} t \sin (t) .
\end{aligned}
$$

where $a$ and $\delta$ are the radius of the inner circle and turns distance respectively. The required derivatives for finding incremental length vector $d l$ in the path of spiral can be expressed as,

$$
\begin{gathered}
x^{\prime}(t)=-a \sin (t)+\frac{\delta}{2 \pi} \cos (t)-\frac{\delta}{2 \pi} t \sin (t) \\
y^{\prime}(t)=a \cos (t)+\frac{\delta}{2 \pi} \sin (t)+\frac{\delta}{2 \pi} t \cos (t)
\end{gathered}
$$




\subsection{Coil geometry - conical}

Disregarding the field leakage and spacing of coils, the parametric equations for conical coils can be derived using the Archimedes equations over the interval $[0,2 n \pi]$.

$$
\begin{gathered}
x(t, a)=a \cos (t) \\
y(t, a)=a \sin (t) \\
z(t, a)=a
\end{gathered}
$$

The normal vector can be derived from basic parametric equations,

$$
\left(\frac{\partial x}{\partial a}, \frac{\partial y}{\partial a}, \frac{\partial z}{\partial a}\right) \times\left(\frac{\partial x}{\partial t}, \frac{\partial y}{\partial t}, \frac{\partial z}{\partial t}\right)
$$

The resultant of cross product is the determinant as usual,

$$
\begin{gathered}
\operatorname{det}\left(\begin{array}{ccc}
P & Q & R \\
\frac{\partial x}{\partial a} & \frac{\partial y}{\partial a} & \frac{\partial z}{\partial a} \\
\frac{\partial x}{\partial t} & \frac{\partial y}{\partial t} & \frac{\partial z}{\partial t}
\end{array}\right)=\operatorname{det}\left(\begin{array}{ccc}
P & Q & R \\
\cos (t) & \sin (t) & 1 \\
-a \cos (t) & a \cos (t) & 0
\end{array}\right) \\
=\left(\begin{array}{ccc}
-a \cos (t), & -a \sin (t), & \left.a \cos ^{2}(t)+a \sin ^{2}(t)\right)
\end{array}\right) \\
=\left(\begin{array}{lll}
-a \cos (t), & -a \sin (t), & a) .
\end{array}\right.
\end{gathered}
$$

Usually, the direction of $z$ component indicates the conical movement either in downward or upward. The positive sign indicates upward and a negative sign indicates downward movement, respectively. Hence, the correct version of normal downward vector is

$$
=(a \cos (t), \quad a \sin (t), \quad-a),
$$

Which is downward facing.

Then the derivatives to solve the Biot-Savart law for obtaining the field intensity of conical coil is as shown in the Eqs. (19.1)-(19.2).

$$
\begin{gathered}
x^{\prime}(t, r)=-a \sin (t) \\
y^{\prime}(t)=a \cos (t)
\end{gathered}
$$

In theory, conical coils are basically a compression of spiral coils increasing or decreasing its outer diameters. This type of coils provides the stability by shrinking the solid height, which means larger slenderness ratio. It also provides downward facing vector lines for an arbitrary point on the cone. Since the nature of downward vector, i.e., high directivity, there is a possibility of reducing power loss in the coupled coils.

\subsection{Finite element simulation}

There are three different source coils such as a solenoid, spiral and conical models are considered as a significant
Table 1. Coil parameters used in the COMSOL simulation.

\begin{tabular}{lc}
\hline Parameters & Value \\
\hline Number of turns & 13 \\
Inner radius (mm) & 3.20 \\
Height (mm) & 242 \\
Inter-turn distance (mm) & 3.20 \\
Wire diameter (mm) & 20 \\
Length (mm) & 20 \\
\hline
\end{tabular}

Table 2. Simulated value of self-inductance of coils.

\begin{tabular}{lccc}
\hline Parameters & Spiral & Solenoid & Conical \\
\hline Self-inductance $(\mu \mathrm{H})$ & 5.87 & 6.54 & 8.63 \\
\hline
\end{tabular}

matter in this work, and respective parameters are listed in tables 1 and 2.

As it is mentioned earlier, with the physical limitation of smaller sized load coil in the receiver section, typically, the diameter of source coil in the transmitter is between 40 and $100 \mathrm{~mm}$, and a number of turns are 5-15 with the coil height of $10-40 \mathrm{~mm}[3,10,11]$. All the three coils are modeled with approximately similar dimensions. A simulation model is developed using COMSOL 4.0 Multiphysics. First, each coil is developed using Solid Works premium edition, then it is simulated using COMSOL simulation platform to extract the characteristics of the magnetic field.

The simulated field pattern of three different source coils such as a solenoid, conical, and spiral geometries are illustrated in figure 5. The coils such as a solenoid in figure 5(a), and spiral in figure 5(b) create the distributive pattern of the magnetic field around the coil. The field intensity is high in the center axis due to the flow of the majority of flux and thereby maximum of flux linkage in this location. It is reduced gradually when moving out from coil center towards its boundary.

Therefore considerable power loss occurred due to the nature of distributed field pattern produced by these coils. But, the field pattern of the conical coil is slightly improved due to its high directivity, which is shown in figure 5(c). In this conical coil, most of the flux lines are taking downward movement and all are gathered together on the coil axis. This cumulative action produces the strong enough magnetic field and flows towards the bottom edge of the coil. If the load coil is placed axially at the bottom of conical shaped source coil, the possibility of maximum flux linkage and better power transfer efficiency could be achieved.

Figure 6 compares the magnetic field behavior of designed coils such as a solenoid, spiral, and conical. It demonstrates the measurement of field pattern at the center axis of all three coils. It can be seen obviously that the 


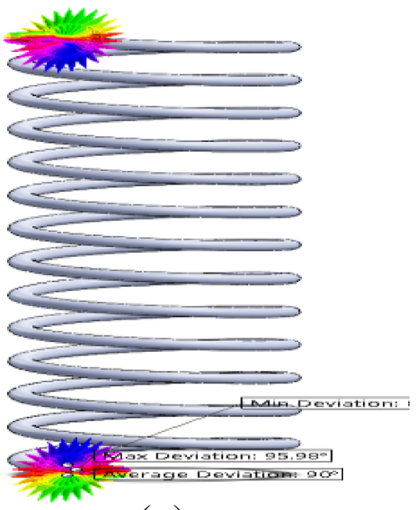

(a)

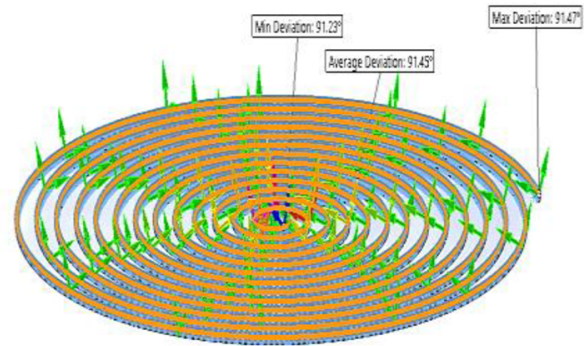

(b)

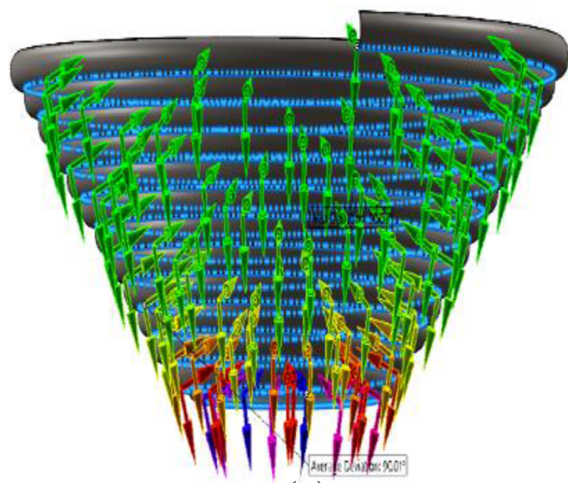

(c)

Figure 5. Simulated field pattern developed coils using COMSOL Multiphysics. (a) Distributive field pattern of the solenoid coil. (b) Distributive field pattern of the spiral coil, and (c) cumulative field pattern of the conical coil.

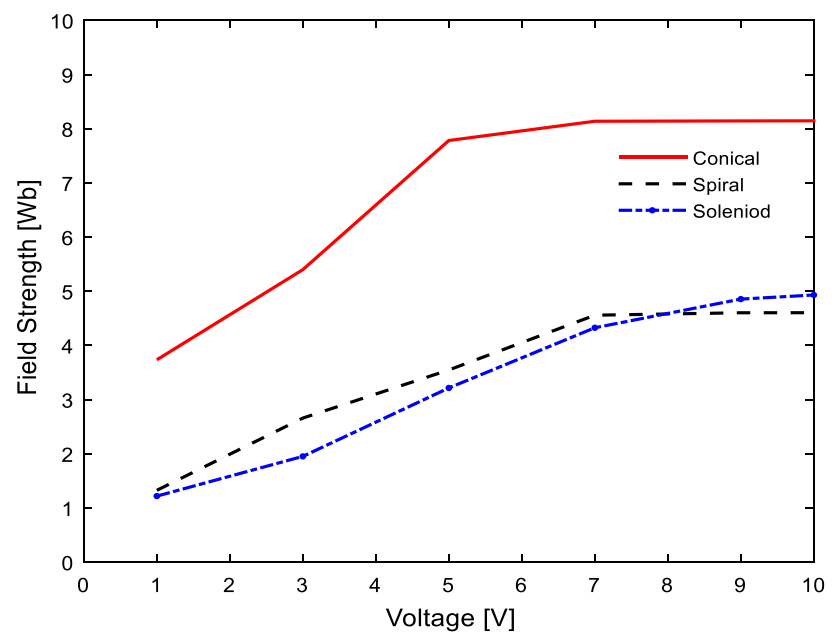

Figure 6. Fundamental parameter analysis of conical, spiral, and solenoid coils as a function of excited dc voltage. Measurement of the magnetic field in the center axis of three different shaped coils.

behavior of spiral and solenoid coils are almost same, but conical performs differently. The measurement has been carried out with the aim of producing a better magnetic field in a single direction instead of having distributed field pattern. The experiments in the previous works focused on producing a distributive pattern and thereby more than $50 \%$ of flux lines are radiated outward of the coil and died out in the form of heat. Hence it is better to redirect most of the flux lines in a particular direction so that maximum field conversion can be accomplished. The plots indicate that the field behavior of conical is good in producing high intensity compared with other two coils. It can be clearly seen that the downward movement nature of conical produces a strong magnetic field at the bottom portion of the coil. It gives the advantage of making use of the high intensity at the bottom side, by which efficient power transfer can be achieved using conical coils. However, conical is oversized than other two coils so that less importance was given in most of the works. Thus, it is not suitable to be used as a load coil in the receiver circuit since the size is limited. In another aspect of using conical as a source coil and spiral as a load coil, it would be a sensible approach for making good power transfer radiation between the conical and spiral coils at the bottom side of conical.

\section{Experiments and analysis}

As it is mentioned earlier, eventually the same circuit configuration of WPT is shared by all designers, but the distinctive way of designing WPT system is desirable. In our work, Matlab-based complete simulation set-up is developed to demonstrate the operation of the inductive link for good transfer efficiency under both aligned and skewed position of coils. Figure 7 illustrates the equivalent transmitter circuit set- up and its flux generation model used in the developed WPT system. It operates based on the fundamental inductive coupling principle. Primarily, the excited dc power is converted into a high-frequency $\mathrm{AC}$ power using full bridge inverter circuit. Then, these highfrequency pulses are applied to excite the coil connected in the transmitter section. Then, the alternating magnetic field is produced across the coupled transmitter coil, induces AC voltage on the receiving coil. The amount of receiving power, losses, and efficiency of the WPT system is mainly dependent on air losses (angle, distance, load resistance value and switching frequency) of the system.

The flux estimation in the transmitter section can be expressed using Eqs. (20)-(21). The flux due to the inverter current can be calculated as,

$$
f l u x(\varphi)=\frac{\tanh \left(\frac{i}{5}\right)}{2}
$$




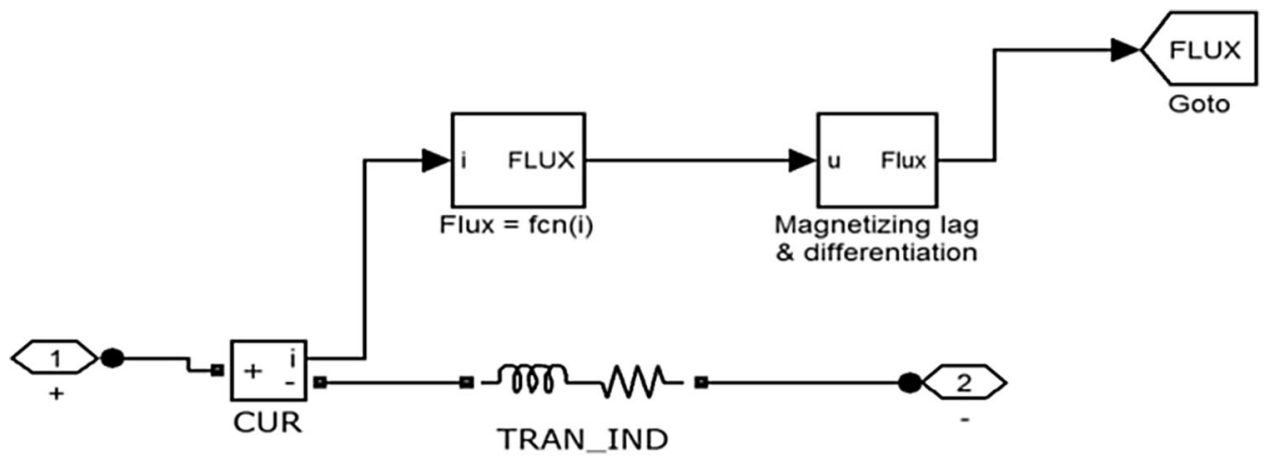

Figure 7. Equivalent model of transmitter circuit used in the WPT system for generating flux from the excited ac pulses at the source coil.

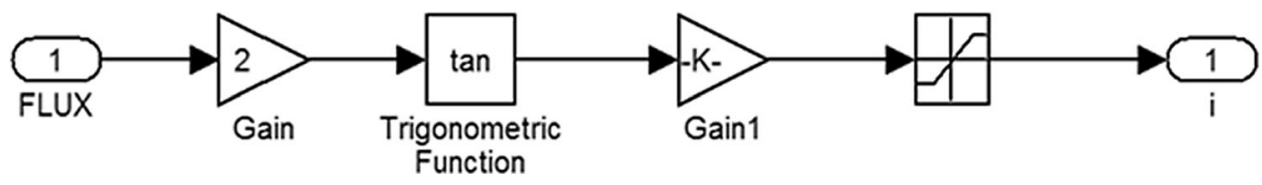

Figure 8. Equivalent model of receiver circuit used in the WPT system for generating current at the load coil from generated flux.

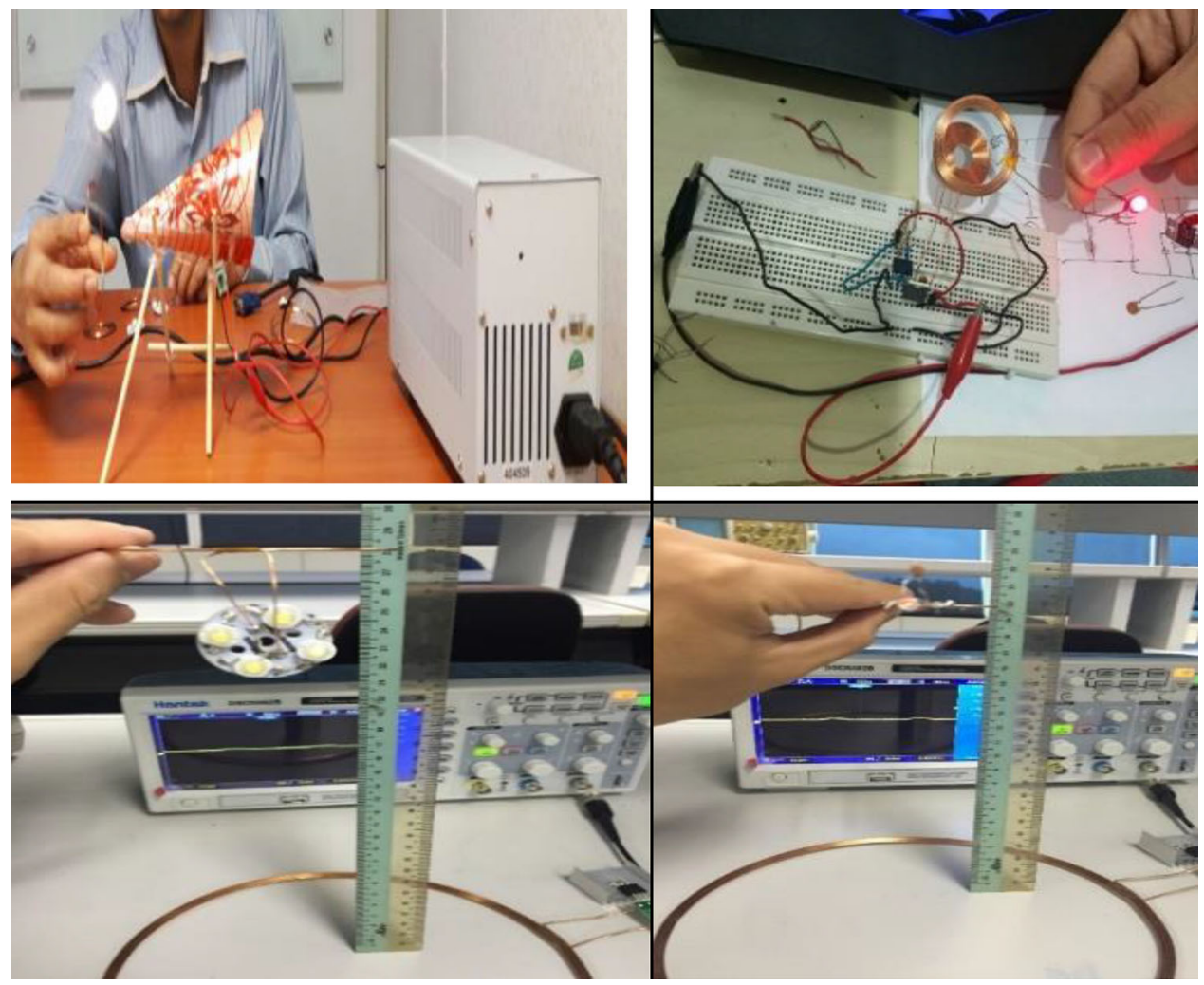

Figure 9. Experimental set-up used for practical measurements.

The estimation of flux derivative in the transmitter coil is,

$$
d f l u x(d \varphi)=\int 2 \pi f_{s} \times\{\varphi(t)-\varphi(t-1)\}
$$

where $t$ and $f_{s}$ represent the $t$ th sampling instance and switching frequency, respectively.

In the receiver section shown in figure 8, the received AC power is converted into dc power using diode bridge 


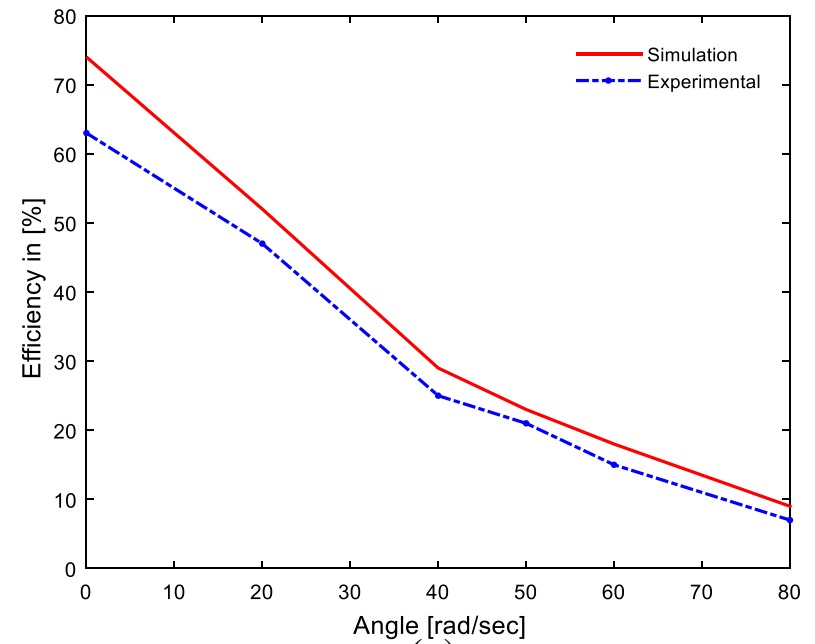

(a)

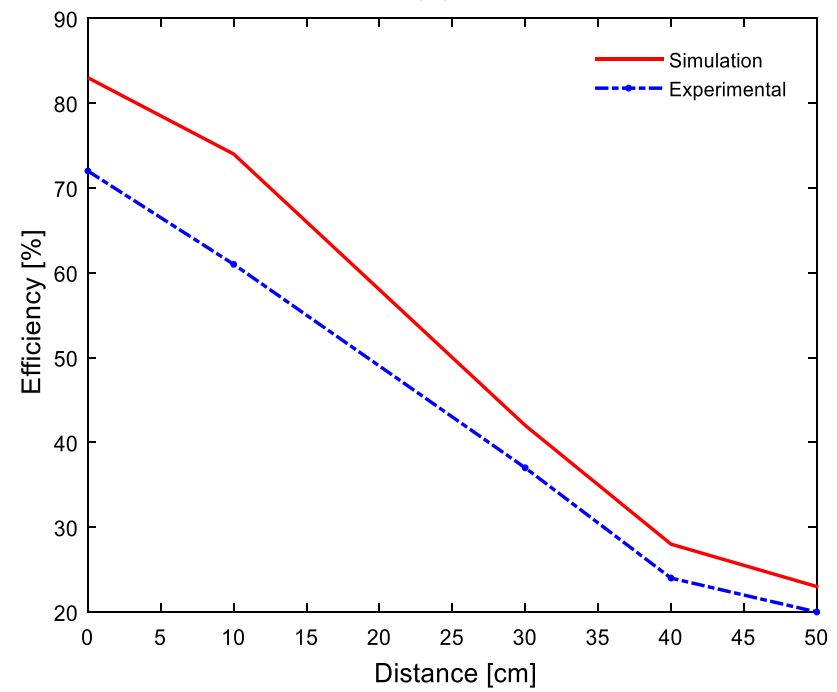

(b)

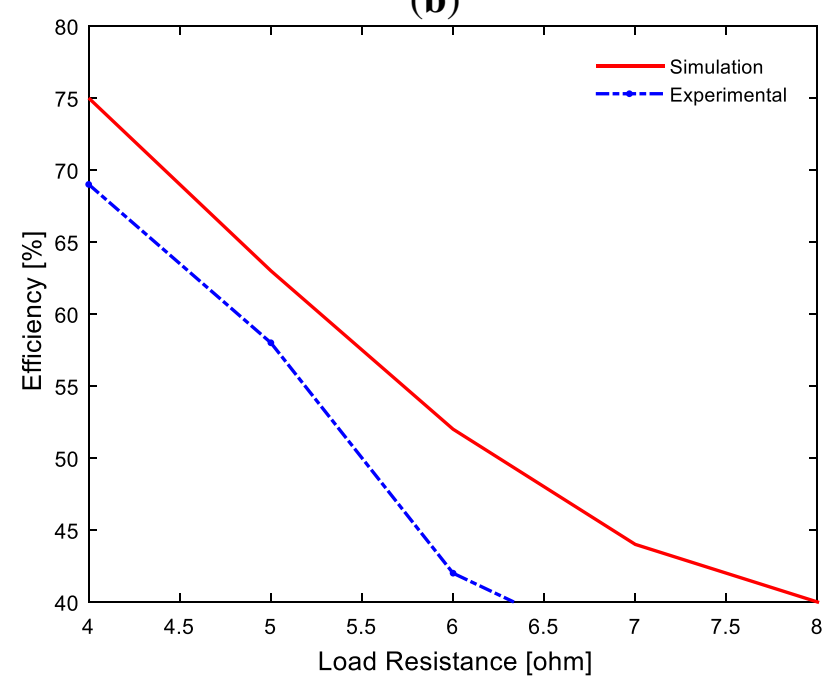

(c)

Figure 10. Power transfer efficiency analysis at $5 \mathrm{MHz}$ switching frequency. (a) Efficiency as a function of angle at a distance of 5 $\mathrm{cm}$. (b) Efficiency as a function of distance at a zero degree angle. (c) Efficiency as a function of the load with a distance of $5 \mathrm{~cm}$. rectifier and then fed into the DC-DC boost converter. The boost converter serves to regulate the output voltage and extract maximum power from the receiving coil according to the Maximum Power Point Tracking (MPPT) algorithm [24].

$$
\text { Receive Power }=\text { Differential flux }- \text { air loss }
$$

Air loss includes the loss due to the load, angle, and distance variation between two coils. The current in the load which is connected to the receiver can be estimated using Eqs. (22) and (23).

$$
\begin{gathered}
\text { flux }(\varphi)=\frac{d(d \varphi)}{d t} \times 2 \pi f_{s}+d \varphi \\
\text { Current }(i)=2 \times \tan \left(\frac{\varphi}{5}\right) .
\end{gathered}
$$

The experiment is conducted in both aligned and misaligned placement of coils, which are illustrated in figure 9 . The graphical plots are shown in figure 10 indicate the variation of three viable parameters involved with power transfer efficiency. The system is tested under $5 \mathrm{MHz}$ switching frequency and $4 \Omega$ load, while distance in-between the coils range from 0 to maximum of $50 \mathrm{~cm}$.

The transmitter coil was excited with dc input voltage and also receiver coil was connected to a resistive load of 4 $\Omega$. The graphical plot is shown in figure 10(a) outlines the variation of transfer efficiency in the case of coils that are not oriented properly (misaligned). The angle is varied from 0 to maximum of 82 degrees with $5 \mathrm{MHz}$ switching frequency and coil separation distance is $5 \mathrm{~cm}$. It is recorded that the maximum efficiency is $75 \%$ initially with an angle of 0 degrees and reduces gradually by increasing the alignment angle. The efficiency is less than $10 \%$ at an angle of 82 degrees. On the other hand, the plot shown in figure 10(b) sketches the variation of transfer efficiency as a function of increasing relative distance between properly aligned coils.

The distance is varied from 0 to maximum of $50 \mathrm{~cm}$ with operating frequency of $5 \mathrm{MHz}$ and zero degrees misalignment.

Initially, it shoots up to the maximum of $82 \%$ and it drops further down, upon increasing the relative distance between coils, and reaches below $30 \%$ when the distance is more than $40 \mathrm{~cm}$. Further, in figure 10(c) the variation of transfer efficiency as a function of load resistance connected with receiver coil is observed. The load resistance is varied from $4 \Omega$ to $8 \Omega$ at a distance of $5 \mathrm{~cm}$ for the properly oriented coils. The value of transfer efficiency reaches $75 \%$ when the load resistance is $4 \Omega$ and it drops further down and reaches a minimum of $40 \%$ at a value of $8 \Omega$. However, it is also observed that there is a substantial difference between simulation and experimental results in the efficiency calculation. This deviation is due to the factors such as accuracy of coil windings, component tolerance levels, coil quality factors, and also coil orientations. But the 
matching between analytical and experimental results are very linear with similar progression. Hence the result analysis matches well and also provides the significant parametrical knowledge in the coil selection for design optimization.

\section{Conclusion}

Power loss in the inductively coupled coils is obvious and few solutions were recommended previously in terms of using multiple source coils, relay coils, near field coupling, resonance coupling, different layered coils, usage of parasitic capacitances, and so on. But, these works did not address completely to the incidences of power loss on the source side. The success of designed WPT system mainly depends on the magnetic field generation of the source coil. The concern on the misalignment of coils is also equally investigated and reported earlier. One to one coil model is not affected much due to the misalignment, but, it is more concern on the presence of multiple load coils.

In this paper, three distinct analyses based on the coil geometry and its parameters are explored for the benefit of realizing a greatly efficient wireless power transfer system. With the limited size and power, it mainly addresses the drawback of power loss occurred in the coil zone, which has highly influenced the coupling between aligned coils. In this paper, the novel conical coil is developed using finite element method based on the parametric equations. As the benefit of downward field patterns produced by conical shape, it is clearly presented that conical coil produces better field strength than those with solenoid and spiral. The significance of source coil geometry is also explored for the benefit of better coupling and power transfer efficiency. In addition, it is proposed to use the MPPT algorithm to implement the WPT system for flux generation and current conversion through inductively.

Furthermore, the experiment has been conducted using uniform coils based on the proposed transmitter and receiver design, as a function of angle, distance and load resistance variation. Finally, the observed results are validated with simulation results with the help of graphical plots. These results confirm our system performance and effectiveness as good in terms of achieving maximum efficiency of $73 \%$ at a distance of $5 \mathrm{~cm}$ under ideal condition. The mouth of the cone coil has more directivity and better field strength. The function of coupled coils of this zone will make a new trend in WPT applications. It will work more effectively in a one-to-one coil scenario and also supports significantly in the multi-coil topology, and misaligned coil configurations. The application of this work finds the usefulness of power transfer into the measuring devices, electrical vehicles and so on.

\section{References}

[1] Schlierf R, Horst U, Ruhl M, Rode T S, Mokwa W and Schnakenberg U 2007 A fast telemetric pressure and temperature sensor system for medical applications. J. Micromech. Microeng. 17(7): 98-102

[2] Ahmadi M M, and Jullien G A X 2009 A wireless-implantable microsystem for continuous blood glucose monitoring. IEEE Trans. Biomed Circuits Syst. 3(3): 169-180

[3] Khan I M, Khan S, and Khalifa O O 2012 Wireless transfer of power to low power implanted biomedical devices coil design considerations. In: Instrumentation and Measurement Technology Conference, vol. 1, pp. 2584-2588

[4] Kurs A, Karalis A, Moffatt R, Joannopoulos J D, Fisher P and Soljacic M 2003 Wireless power transfer via strongly coupled magnetic resonances. Science 80: 37-46

[5] Kurs A and Lestoquoy G 2015 A framework for evaluating multi-kilowatt highly-resonant wireless power transfer systems. In: IEEE 82nd Vehicular Technology Conference (VTC Fall), Boston, MA, USA

[6] Huh J, Lee S W, Lee W Y, Cho G H and Rim C T 2011 Narrow-width inductive power transfer system for online electrical vehicles. IEEE Trans. Power Electron. 26(12): 3666-3679

[7] Budhia M, Covic G A and Boys J T 2011 Design and optimization of circular magnetic structures for lumped inductive power transfer systems. IEEE Trans. Power Electron. 26(11): 3096-3108

[8] Nataraj C, Khan S, Habaebi M H, A G A Muthalif and R Lakshmanan 2017 Development of low power wireless power transfer system using resonance principle with security features. IIUM Eng. J. (IIUMEJ) 18(2):117-127

[9] Van Thuan N, Kang S H, Choi J H, Jung C W 2015 Magnetic resonance wireless power transfer using three-coil system with single planar receiver for laptop applications. IEEE Trans. Consum. Electron. 61(2): 160-166

[10] Bosshard R, Muhlethaler J, Kolar JW, and Stevanovic I 2013 Optimized magnetic design for inductive power transfer coils. In: Twenty Eighth Annual IEEE Applied Power Electronics Conference and Exposition, Long Beach, CA, USA

[11] Torla A L, Khan S, Muthalif A G, Azman A W and Mustafah Y M 2013 Low voltage DC power supply with spikeblocking features. In: IEEE International Instrumentation and Measurement Technology Conference (I2MTC), Minneapolis, MN, USA

[12] Nguyen M Q, Hughes Z, Woods P, Seo Y-S, Rao S, Chiao J-C 2014 Field distribution models of spiral coil for misalignment analysis in wireless power transfer systems. IEEE Trans. Microw. Theory Tech. 64(4): 920-930

[13] Matias R, Cunha B and Martins R 2013 Modeling of inductive coupling for wireless power transfer to integrated circuits. In: IEEE Wireless Power Transfer, Perugia, Italy

[14] Hwang H, Moon J, Lee B, Jeong C-H and Kim S-W 2014 An analysis of magnetic resonance coupling effects on wireless power transfer by coil inductance and placement. IEEE Trans. Consum. Electron. 60(2): 203-209

[15] Bosshard R, Badstuibner U, Kolar J W and Stevanovic I 2012 Comparative evaluation of control methods for inductive power transfer. In: International Conference on Renewable Energy Research and Applications, Nagasaki, Japan 
[16] Ng W M, Zhang C, Lin D, and Ron S Y 2014 Two- and three-dimensional omnidirectional wireless power transfer. IEEE Trans. Power Electron. 29(9): 4470-4474

[17] Pantic Z, Lee K, and Lukic S M 2015 Receivers for multifrequency wireless power transfer: Design for minimum interference. IEEE J. Emerg. Sel. Top. Power Electron. 3(1): 234-241

[18] Fotopoulou K, Flynn B W 2011 Wireless power transfer in loosely coupled links: coil misalignment model. IEEE Trans. Magn. 47(2): 416-430

[19] Sun T, Xie X, Li G, Gu Y, Deng Y, Wang Z 2012 A two-hop wireless power transfer system with an efficiency-enhanced power receiver for motion-free capsule endoscopy inspection. IEEE Trans. Biomed. Eng. 59(11):3247-3254

[20] Qiang H, Zhu W and Zheng J 2013 Simulation study on coil design of wireless power transfer system for optimal transmission efficiency. Res. J. Appl. Sci. Eng. Technol. 5(21): 5039-5041
[21] Johari R, Krogmeier J V, and Love D J 2014 Analysis and practical considerations in implementing multiple transmitters for wireless power transfer via coupled magnetic resonance. IEEE Trans. Industr. Electron. 61(4): 1174-1783

[22] Nataraj C, Khan S, Habaebi M H and Muthalif A G A 2016 Resonant coils analysis for inductively coupled wireless power transfer applications. In: IEEE International Instrumentation and Measurement Technology Conference, Taipei, Taiwan

[23] Nataraj C, Khan S, Habaebi M H and Muthalif A G A 2016 Hybrid of Conical and Spiral Approach for Wireless Power Transfer. In: IEEE Student Conference on Research and Development (SCOReD), Kuala Lumpur, Malaysia

[24] Dzung P Q, Le Dinh K, Lee H H, Phuong L M and Vu N T D 2010 The New MPPT Algorithm Using ANN-Based PV. In: IEEE International Forum on Strategic Technology (IFOST), Ulsan, Korea (South) 